\title{
On-Line Tools for Management of Multi-user Microscopy Facilities
}

\author{
D-M Zheng,* M Stevens-Kalceff,* and P.R Munroe* \\ * Electron Microscope Unit, University of New South Wales, SYDNEY, NSW2052, Australia
}

It is increasingly common for government funded organizations to co-locate research infrastructure into central facilities. Instrumentation is increasingly sufficiently complex and expensive that institutions insist that the equipment is shared through central management. There are a number of advantages of this approach. Not least, that this gives provision for a critical mass of staff and the equipment is more highly utilized, since it is shared across a larger number of users. Parallel to such a trend is the requirement for greater accountably, more stringent requirements associated with occupational health and safety, in particular through the establishment of records for training and risk assessment, and, further, the need to levy charges on users. Many of these large centralized facilities provide research services to many hundreds of users across a range of departments, other institutions and commercial clients. The day-to-day management requires complex systems to control and share instrument access, capture usage and communicate with users.

The Electron Microscope Unit (EMU) at the University of New South Wales operates as such a central facility. This Unit operates 15 frontline microscopes, together with extensive preparation equipment, and employs 15 staff members. Each year around 400 separate projects are supported and the Unit provides around 20,000 beam hours. Many of the facility users are domiciled at other institutions or with private industries. The Unit is, itself, part of a campus wide Analytical Centre (AC) which includes similar units for NMR, x-ray diffraction, mass spectroscopy etc.

In response to the larger number of users and diverse range on instruments and projects, staff at the EMU has generated the 'AC Lab System' as a tool for operational management of the Unit.

The AC Lab System runs under a Windows operating system server to provide web-based lab information, bookings, sample tracking and management platform to the management, staff and users of the facility. ACLS was developed through Delphi and PHP, and works with a Delphi BDE database engine (this will be upgraded to SQL database engine in the future). The web-based component is built to provide one compact DLL (dynamic link library) to work with the web server. Since the DLL is loaded to the server memory, it enhances response speed and makes system maintenance and upgrades very simple. The system is compatible with all major internet browsers.

A new user to the facility is first required to go through an induction process with a Unit staff member. At this meeting the user is given an OHS induction, there is discussion on the objectives of the microscopy to be performed and training sessions are scheduled. In addition, the user provides their contact and project details and, for usage charges, billing details. For most researchers this is an internal project account code. This data is entered online into a number of data fields.

Initially, (untrained) users have limited access to the system. Users are classified as trainees (category A), independent (category B), with free access to an instrument during normal business hours, or expert (category C), with 24-7 instrument access. Users must pass a driving test type examination, against defined competency criteria to advance their status. The system is designed so 
that EMU staff issue on-line "certificates" to users as they progress in expertise. When users make on-line instrument bookings they can only book that instruments for which they hold certificates, and for time periods consistent with their level of expertise. Thus, a user may hold category $\mathrm{C}$ status for a SEM and may book a SEM at weekends, but only category B status for a TEM and have access only for normal business hours. These training "certificates" then provide and archive a papertrail recording user indication and any associated OHS training.

When a user performs microscopy, they must first login into the ACLS. For users with several projects, and several project accounts, the can select through a drop down menu the account number to be charged for this project. This login acts as to authenticate the user, and login requests by untrained or invalid users are rejected. At the end of the session the user logs out. The exact usage time is calculated. Users are billed for their time of usage and at the end of the billing cycle (monthly) the total usage is compiled directly for each user of each instrument, through an Excel spreadsheet. This makes the administrative calculation of user charges completely automatic. The usage data is readily sorted by field, so that facility managers can, within a few seconds, sort and analyse usage by instrument, user, department, project etc.

Users themselves cannot not only book usage, but access their data through the ACLS, stored in a central server and monitor their own usage. Users can also themselves update their own project details online. Almost all instrument data is stored digitally. This is archived monthly. The current data archive includes all data stored in the Unit since 1997.

Staff and senior management of the Unit have higher levels of access. Technical staff are able to issue training certificates, as described above and automatically schedule reminder emails to users with upcoming training sessions. Moreover, they have the ability to "service book" instruments that are taken off line due to routine maintenance or unexpected failure. This prevents new bookings by users and automatically sends emails to those users with bookings explaining their booking has been cancelled due to instrument downtime.

Unit managers have further access privileges, which allow them to control individually access of users to particular instruments. For example, the hourly usage charge for each user or access to a particular instrument can be individually varied. The time periods for which different users can access different instruments can also be individually tailored. For example, users may book longer periods on instruments such a microprobe, which requires long sessions, but are restricted to shorter sessions on more popular instruments.

The AC Lab system has been developed over a period of around 10 years at UNSW. It is continually upgraded and developed to meet changes, as higher degrees of accountability in running central facilities is required. Furthermore, this system has now been installed and operates at a number of other similar facilities not only throughout Australia, but also in Singapore and Ireland. 